\title{
UNCONSTRAINED HEALTH MONITORING AND EFFECTIVE POSITION TRACKING USING WIRELESS SENSOR NETWORKS FOR CONSTRUCTIONAL LABOURERS
}

\author{
K.Praveenkumar ${ }^{1}$, S.Nivas Ramnath ${ }^{2}$ \\ ${ }^{I}$ B.E, Electronics and Communication Engineering, Mepco Schlenk Engineering College, Sivakasi, India \\ ${ }^{2}$ B.E, Electronics and Communication Engineering, Mepco Schlenk Engineering College, Sivakasi, India
}

\begin{abstract}
The paper introduces a wireless sensor network platform specifically designed for the protection of workers employed in the building sector. The network configuration makes use of GSM communication which provides a more efficient and long range wireless transmission. The system has been developed to determine two different kinds of information, heart beat rate and latitude and longitude corresponding to the location of each worker. These data provide a periodic update of both health and position inside their working environment by sending SMS to higher officials. The sensor node is embedded on the worker garment by integrating within the fabric which forms a unique structure. This system provides a secure environment for the safety of constructional workers by the real-time monitoring of their working surface.
\end{abstract}

Keywords-GSM communication; Wireless Sensor Netowrks; Real-time monitoring; safety.

\section{INTRODUCTION}

Wireless Sensor Networks (WSNs) are used in many technological areas, including environment monitoring, health care applications, logistic, industrial automation and management. They are particularly efficient in situations when cables cannot be used or sensors are in mobility. Among all possible applications, the monitoring of physical phenomena by means of small, non invasive devices represents a challenge for the next future.

In this paper a wireless sensor network is implemented, to monitor the safety conditions of workers employed in the building sectors where accidents occur without any notice [1]. These people are potentially at risk due to harsh climatic conditions or occurrence of any natural calamities. Uneven climatic conditions could result in high blood pressure or reduced heart rate. Hence periodic health monitoring should be done to reduce accidents in the working area [2]. The system can be altered for developing wireless sensor network for monitoring workers in coal mine safety environment [3]. This system can also act as a base for life saving of workers in case of any disaster by finding their location in the accidental area.

\section{WSN ARCHITECTURE}

The network architecture is composed of mobile sensing units, GSM shield and magnetic compass. The sensor node consisting of pulse sensor, microcontroller, GSM shield and magnetic compass must accompany the worker during the whole day, and for this reason it must be wearable, light in weight, small, robust as possible, but also extremely cheap. The sensor node is used to detect the physical phenomenon, process it, obtain result and transmit it to the specified destination. The link among the sensor nodes and the destination is realized with the different methods, depending on the application.

The sensor nodes employed in this application is based on the large outdoor environment and hence GSM standard is preferred. With GSM communication, it is possible to cover large distance of data transmission in a feasible way. The microcontroller collects data from both sensor and magnetic compass and sends the information corresponding to their health status and their location through SMS to reach long distances with less power.

The destination receives SMS from all sensor nodes with their respective GSM number independently. In case the sink wishes to obtain information about the source, reverse process is carried out by making a call to the corresponding GSM number of the source. 

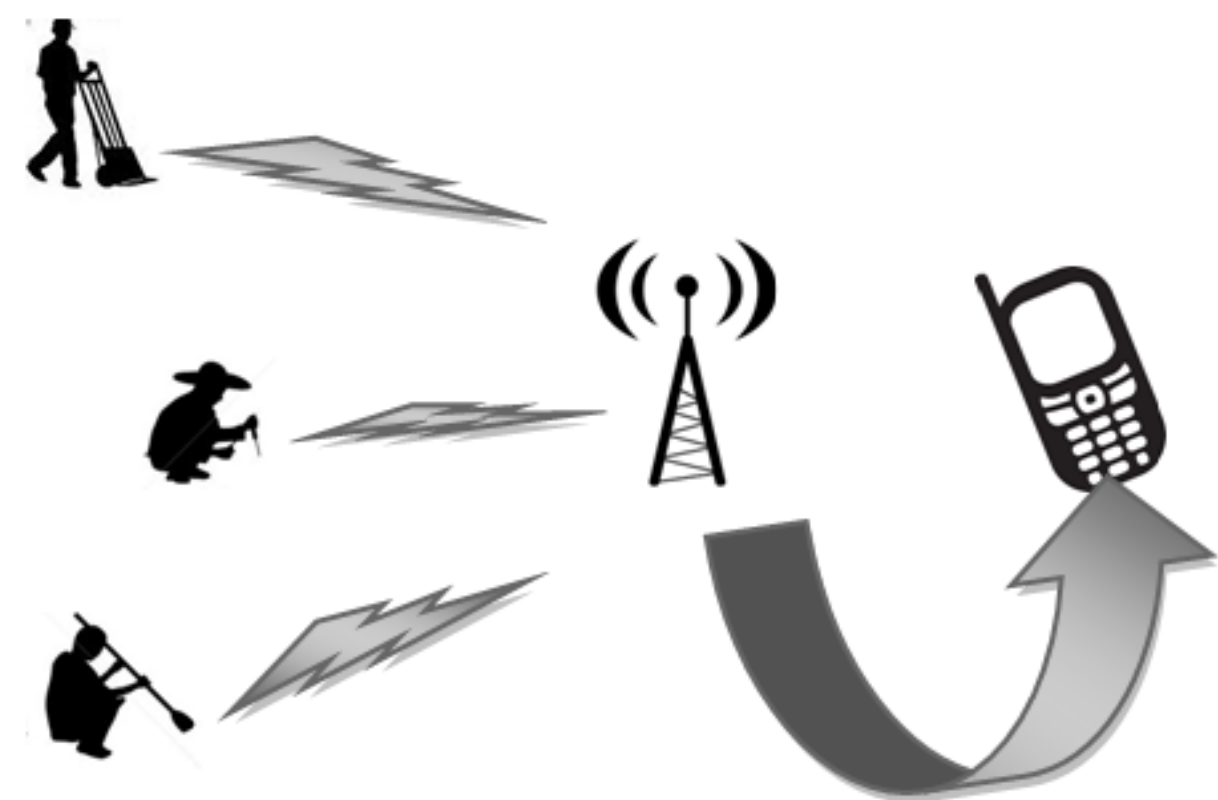

Fig. 1 Wireless Sensor Network scheme: devices are worn by the builders, independently on their working duty, GSM shield collect and transmit information by standard communication.

\section{SENSOR NODE}

The core of WSN is the sensor node that must be designed according to the specification reported in section II. It is composed of:

- a pulse sensor used to detect the heart beat rate,

- a magnetic compass to determine the height,

- a GSM shield to determine the latitude and longitude of the location and to send the information through SMS,

- a battery,

- a microcontroller to process data obtained from sensor and compass.

- a suitable energy harvester able to work in outdoor location.

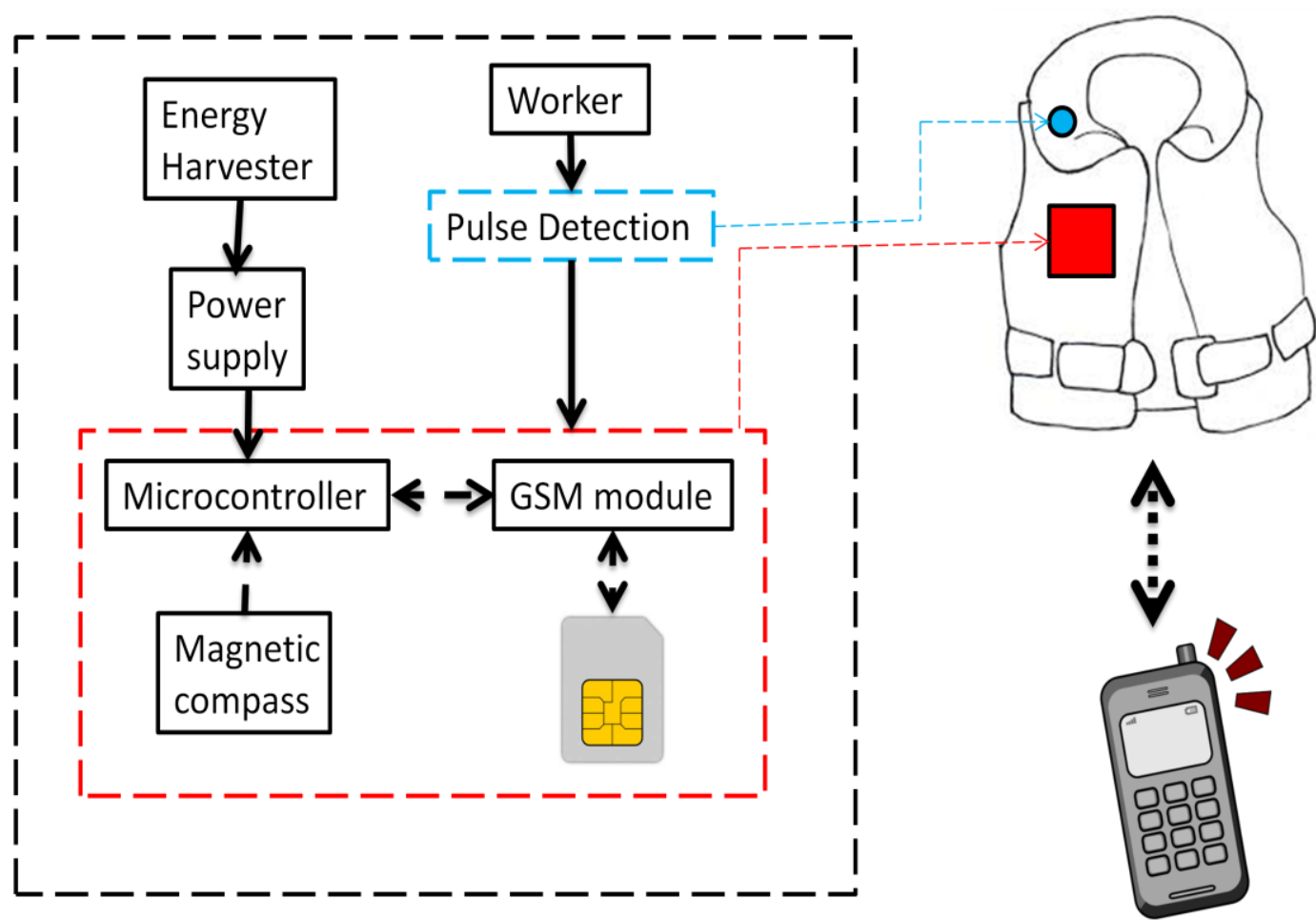

Fig. 2 Sensor Node scheme. The device composed of an harvesting part, which can be realized either with a microsolar panel or a thermoelectric generator, a microcontroller, a GSM shield, a magnetic compass and a pulse sensor. 


\section{PULSE SENSOR}

Among all possible pulse sensing techniques we have chosen an open source hardware project named, Pulse sensor Amped, since this kind of sensor is small, with a high speed and with a good spectral efficiency. We have chosen the SEN-11574 pulse sensor that is effective in detecting heart beat pulses. This sensor makes use of the ambient light sensor from Avago (APDS-9008) and the green super bright reverse mount LED from Kingbright (AM2520ZGC09). It has a current consumption of 4mA at an input voltage of $5 \mathrm{~V}$. The pulse sensor adds diode protection on the power, So it can't break if it is plugged in backwards, and an active filter to make the pulse waveform brighter and easy for the microcontroller to find.

For the pulse sensor, the structure is circular with a diameter of $16 \mathrm{~mm}$ and the additional components makes an increase in size a little to $5 / 8$ " diameter and hence the overall thickness this $3 \mathrm{~mm}$. When the pulse sensor is in close contact with the body the change in reflected light when blood pumps through tissues makes the signal fluctuate and decides a pulse is found when the signal rises above the specific voltage.

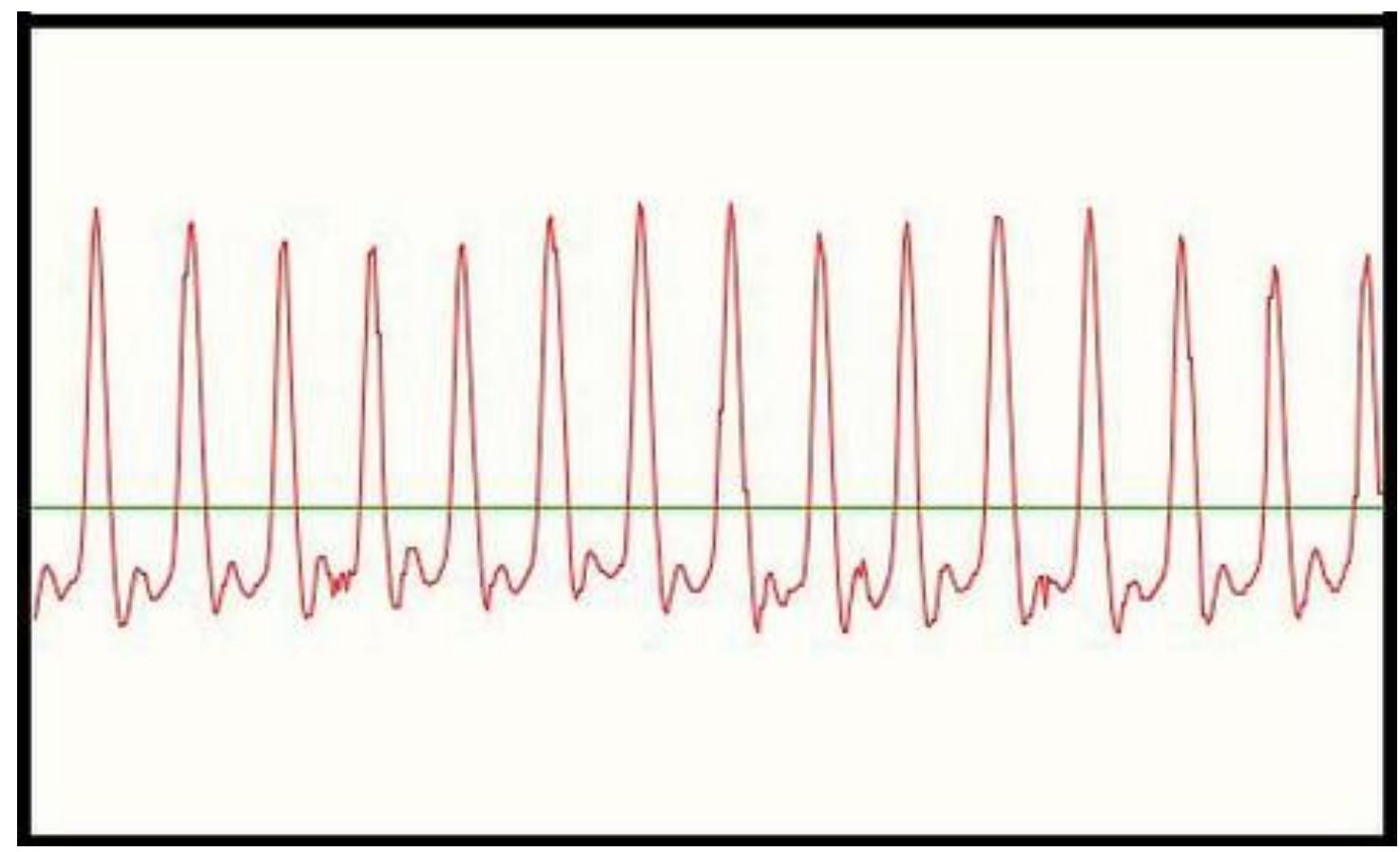

Fig. 3 Output of pulse sensor where the waveform amplitude goes above the reference point and below the horizontal line is V/2.

\section{MAGNETIC COMPASS}

For an effective tracking of location, the determination of height is considered as one of the important factor and this height identifies the storey on which the sensor node is located. Depending on the environmental factors, we decided to use HDPM01 which is an integrated magnetic compass that gives the absolute values of pressure and temperature. The HDPM01 pressure module includes a piezo-resistive pressure sensor and an ADC interface. It provides 16 bit word data for pressure and temperature related voltage. With the help of a highly accurate calibration of the senor, 11 unique coefficients were stored on the chip, thus accurate pressure and temperature reading can be realized.

\section{MICROCONTROLLER AND GSM MODULE}

The processing of data read by the pulse sensor and magnetic compass is carried out in the microcontroller (Arduino Uno Rev 3). The analog waveform form the pulse sensor is serially read in the microcontroller at the baud rate of 9600 . Based on the amplitude of the analog waveform, the pulse rate is measured by counting the number of times the amplitude of the waveform cross the horizontal reference point. In general, for an adult the heart rate should be obtained in the range from 60 to 100 . Hence by identifying the heart rate, the decision is made by the microcontroller by measuring the pulse count and to determine the health status of the worker. The magnetic compass is interface with the microcontroller based on I2C interfacing. The controller also obtains information about the latitude and longitudinal location and transmits through SMS to corresponding destination.

\section{GSM SHIELD}

GSM/GPRS shield provides a hassle free solution to connect the microcontroller to the cellular network. The shield is based on Dual band SIM900A GSM module. The shield uses digital pins for $\mathrm{Rx}$ and $\mathrm{Tx}$ for software serial communication with the SIM900A. It supports TCP/UDP and HTTP protocols through a GPRS connection. The control over the GSM module is carried out using AT commands and it can be powered with an external power supply that can provid current upto 2 Amp. 


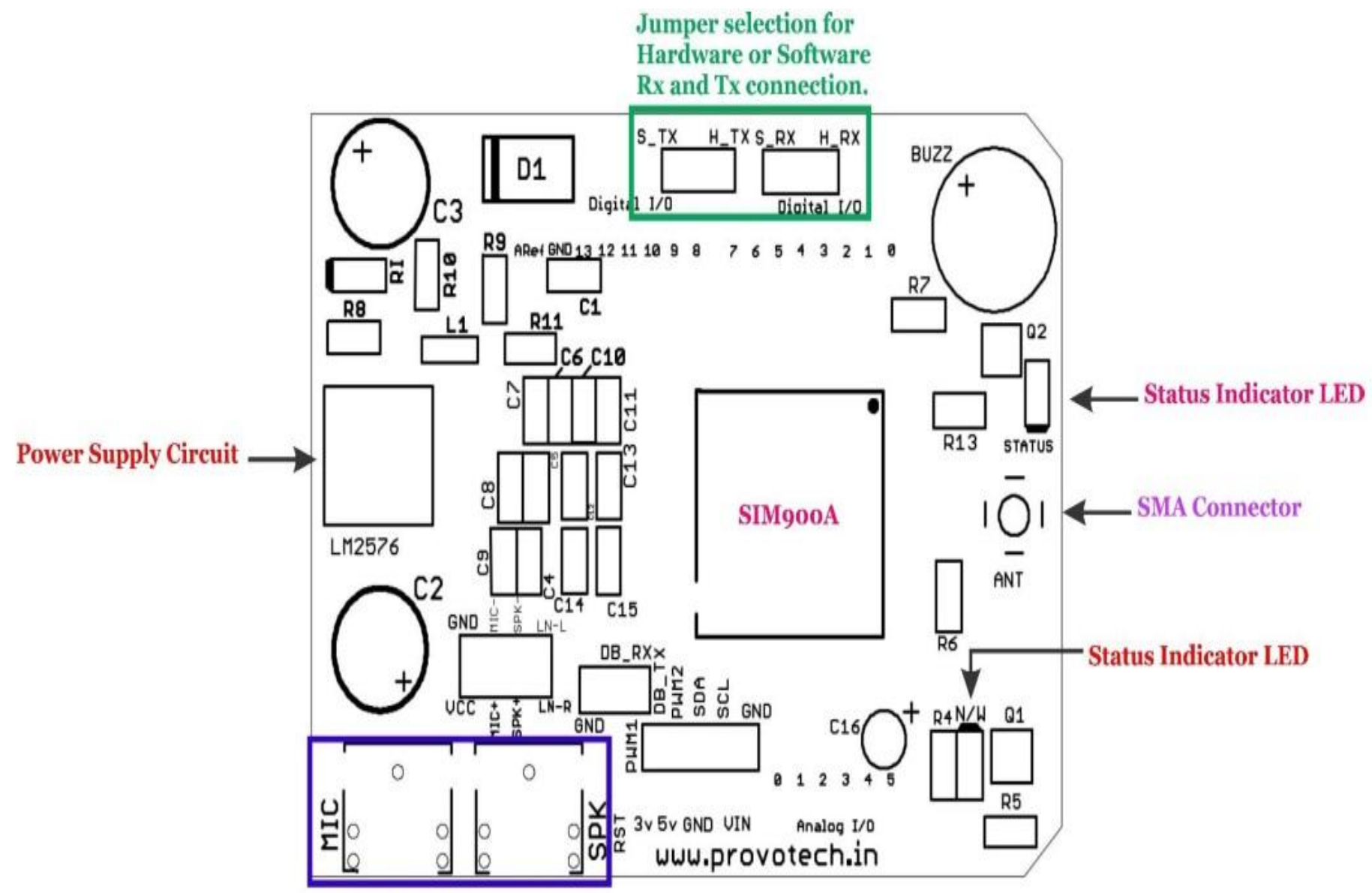

Audio Jack for connecting external MIC and Speaker.

Fig. 4. Layout of the GSM shield. The device is configured to make jumper connection for Tx and Rx and powered for standard GSM communication.

\section{ENERGY HARVESTING}

Since the $\mathrm{SN}$ has to be auto-rechargeable, one important aspect to take into account is the power supply. All SN components are chosen in order to minimize power consumption, but an energy harvester is added to extend the operating life of the sensor.

Different types of Energy harvesters were analyzed [4], and two were found suitable for the project: a thermoelectric one, applicable to indoor locations, and a photovoltaic one, applicable outdoor.

The thermoelectric harvester (Micropelt, MPG D751) is based on the Seebeck effect, with the hot side of the device in contact with the human skin and the cold one exposed to the external environment. The difference of temperature between the bodies, normally larger than $10^{\circ} \mathrm{C}$, is sufficient to generate electrical energy and charge the battery.

The photovoltaic harvester (Ixys, SolarMD600H1OL) is optimal in outdoor construction premises, as the situation is typically favorable to maximize the efficiency of the solar modules.
The energy harvesters are interfaced with the batteries (Infinite Power Solution, Thinergy MEC 201) by means of a dedicated charging circuit (Maxim, MAX17710) that controls the state of the battery and that allows the current flow when necessary.

\section{CONCLUSION}

The paper presents the configuration scheme and subsequent realization of a wearable device suitable to monitor working conditions of builders engaged in tough locations. The current prototype is not optimized in dimensions. For the moment it has been realized making use of development boards, instead of the single components. Nevertheless, the preliminary tests demonstrate the applicability of the concept. In particular, the chosen manufacturing process is reliable, efficient, inexpensive, and allows a general protection to the device, allowing also its washability. 


\section{REFERENCES}

[1] Lynch J Pand Kenneth J L, "A summary revies of wireless sensors and sensor netwoks for structural health monitoring[J]- The Shock and Vibration Digest, 2006, 38(2): 91-128.

[2] ZhaoMing and Wang Junwei, Wireless Sensing System for Civil Structure Construction Monitoring[J], Journal of Electronic Measurement and Instrument, Vol.22,No.6,2008,pp.117-121.

[3] Yu Lin-min, Lin Anqi, Sun Zheng and Li Hui, "Design of Monitoring System for Coal Mine safety Based on Wireless Sensor Network," Mechtronic and Embedded Systems and Applications Beijing, IEEE/ASME International Conference on, 12-15 Oct. 2008.

[4] H. Adnan, "Energy harvesting: State-of-the-art," Renewable Energy, pp. 2641-2654, October 2011. 